\title{
On the oxygen abundance determination in HII regions. High-metallicity regions
}

\author{
L. S. Pilyugin \\ Main Astronomical Observatory of National Academy of Sciences of Ukraine, Goloseevo, 03680 Kiev-127, Ukraine
}

Received 26 October 2000 / Accepted 22 December 2000

\begin{abstract}
This is our second paper devoted to the problem of line intensity - oxygen abundance calibration starting from the idea of McGaugh (1991) that the strong oxygen lines ([OII] $\lambda \lambda 3727,3729$ and $[\mathrm{OIII}] \lambda \lambda 4959,5007)$ contain the necessary information to determine accurate abundances in HII regions. In the previous study (Pilyugin 2000) the corresponding relations were obtained for the low-metallicity HII regions $(12+\log \mathrm{O} / \mathrm{H} \leq 7.95$, the lower branch of the $\mathrm{O} / \mathrm{H}-R_{23}$ diagram). The high-metallicity $\mathrm{HII}$ regions $(12+\log \mathrm{O} / \mathrm{H} \geq 8.2$, the upper branch of the $\mathrm{O} / \mathrm{H}-R_{23}$ diagram) are considered in the present study. A relation of the type $\mathrm{O} / \mathrm{H}=f\left(P, R_{23}\right)$ between oxygen abundance and the value of abundance index $R_{23}$, introduced by Pagel et al. (1979), and the excitation parameter $P$ (which is defined here as the contribution of the radiation in [OIII] $\lambda \lambda 4959,5007$ lines to the "total" oxygen radiation) has been derived empirically using the available oxygen abundances determined via measurement of a temperature-sensitive line ratio [OIII]4959,5007/[OIII]4363 ( $T_{\mathrm{e}}$-method). By comparing oxygen abundances in high-metallicity HII regions derived with the $T_{\mathrm{e}}$-method and those derived with the suggested relations $(P$-method), it was found that the precision of oxygen abundance determination with the $P$-method is around 0.1 dex (the mean difference for the 38 HII regions considered is $\sim 0.08$ dex) and is comparable to that of the $T_{\mathrm{e}}$-method. A relation of the type $T_{\mathrm{e}}=f\left(P, R_{23}\right)$ between electron temperature and the values of abundance index $R_{23}$ and the excitation parameter $P$ was derived empirically using the available electron temperatures determined via measurement of temperature-sensitive line ratios. The maximum value of differences between electron temperatures determined via measurement of temperature-sensitive line ratios and those derived with the suggested relation is around $1000 \mathrm{~K}$ for HII regions considered here, the mean value of differences for $38 \mathrm{HII}$ regions is $\sim 500 \mathrm{~K}$, which is the same order of magnitude as the uncertainties of electron temperature determinations in high-metallicity HII regions via measured temperature-sensitive line ratios.
\end{abstract}

Key words. ISM: HII regions - galaxies: abundances - galaxies: ISM - galaxies: spiral

\section{Introduction}

An investigation of chemical properties of galaxies is very important for the development of the theory of structure and evolution of galaxies. Oxygen plays a key role in understanding the (chemical) evolution of galaxies for a several reasons. Firstly, good spectrophotometry of HII regions is available now for a large number of galaxies, and the oxygen abundances derived from line intensities are published in many works (Caplan et al. 2000; Deharveng et al. 2000; Esteban et al. 1998, 1999a,b; Garnett et al. 1997; Izotov \& Thuan 1998, 1999; Izotov et al. 1994, 1997; Kobulnicky \& Skillman 1996, 1997, 1998; Kobulnicky et al. 1997; Skillman et al. 1994; van Zee et al. 1997, 1998; among others). Secondly, the origin of oxygen seems to be reliably established in contrast to other elements like carbon or nitrogen. The oxygen abundance can be considered as a tool to investigate the evolution of galaxies.
For example, the value of oxygen abundance in a galaxy combined with the value of the gas mass fraction can tell us about the efficiency of mass exchange between a galaxy and its environment (Pilyugin \& Ferrini 1998, 2000).

Accurate oxygen abundances can be derived from measurement of temperature-sensitive line ratios, such as [OIII] 4959,5007/[OIII]4363. This method will be referred to as the $T_{\mathrm{e}}$-method. Unfortunately, in oxygen-rich HII regions the temperature-sensitive lines such as [OIII] 4363 are too weak to be detected. For such HII regions, empirical abundance indicators based on more readily observable lines were suggested (Pagel et al. 1979; Alloin et al. 1979). The empirical oxygen abundance indicator $R_{23}=([\mathrm{OII}] 3727,3729+[\mathrm{OIII}] 4959,5007) / \mathrm{H}_{\beta}$, suggested by Pagel et al. (1979), has found widespread acceptance and use for the oxygen abundance determination in HII regions where the temperature-sensitive lines are undetectable. This method will be referred to as the

\footnotetext{
* e-mail: pilyugin@mao.kiev.ua
} 
$R_{23}$-method. Several workers have suggested calibrations of $R_{23}$ in terms of oxygen abundance (Edmunds \& Pagel 1984; McCall et al. 1985; Dopita \& Evans 1986; Zaritsky et al. 1994, among others).

There are two problems with the oxygen abundances derived by the $R_{23}$-method. First, oxygen abundances derived with different $R_{23}$-calibrations can differ by 0.3 dex and more. The usually-used $R_{23}$ calibrations (Edmunds \& Pagel 1984; McCall et al. 1985; Dopita \& Evans 1986; McGaugh 1991) are based on a few then-available oxygen abundance determinations through the $T_{\mathrm{e}}$-method and HII region models. More oxygen abundance determinations through the $T_{\mathrm{e}}$-method are available now. None of these $R_{23}$ calibrations can reproduce the available data well enough (Pilyugin 2000, Paper I). Secondly, it has been found (Paper I) that the error in the oxygen abundance derived with the $R_{23}$-method involves two parts: the first is a random error and the second is a systematic error depending on the excitation parameter. The origin of this systematic error is as follows. In a general case the intensities of oxygen emission lines in spectra of HII regions depend not only on the oxygen abundance but also on the physical conditions (hardness of the ionizing radiation and geometrical factor). Then in the determination of the oxygen abundance from line intensities the physical conditions in the HII region should be taken into account. In the $T_{\mathrm{e}}$-method this is done via $T_{\mathrm{e}}$. In the $R_{23}$-method the physical conditions in an HII region are ignored.

In our recent work (Paper I), a new way of oxygen abundance determination in HII regions $(P$-method) was suggested. A more general relation of the type $\mathrm{O} / \mathrm{H}=$ $f\left(P, R_{23}\right)$ is used in the $P$-method, compared to the relation of the type $\mathrm{O} / \mathrm{H}=f\left(R_{23}\right)$ used in the traditional $R_{23}$-method. It was found in Paper I that the precision of oxygen abundance determinations in low-metallicity $(12+\log \mathrm{O} / \mathrm{H} \leq 7.95) \mathrm{HII}$ regions with the $P$-method is comparable to that with the $T_{\mathrm{e}}$-method. The oxygen abundances of high-metallicity $(12+\log \mathrm{O} / \mathrm{H} \geq 8.2) \mathrm{HII}$ regions derived with the $P$-method are significantly less accurate. This seems to be because a subset of HII regions with high-quality homogeneous determinations of oxygen abundances was used in the construction of the $\mathrm{O} / \mathrm{H}=$ $f\left(P, R_{23}\right)$ relation in the case of the low-metallicity HII regions while the relation for high-metallicity HII regions was based on the set of all available HII regions with inhomogeneous determinations of oxygen abundances. Here the oxygen abundances for high-metallicity HII regions with measured line ratios $[\mathrm{OIII}] \lambda \lambda 4959,5007 / \lambda 4363$ will be recomputed in a uniform manner, and the set of HII regions with homogeneous determinations of oxygen abundances will be used in the construction of the $\mathrm{O} / \mathrm{H}=$ $f\left(P, R_{23}\right)$ relation in the case of the high-metallicity HII regions.

The search for an $\mathrm{O} / \mathrm{H}=f\left(P, R_{23}\right)$ relation for highmetallicity HII regions is the goal of this study. The preliminary analysis of the relevant observational data and redetermination of oxygen abundance in a uniform way for a large set of HII regions are given in Sect. 2. The line

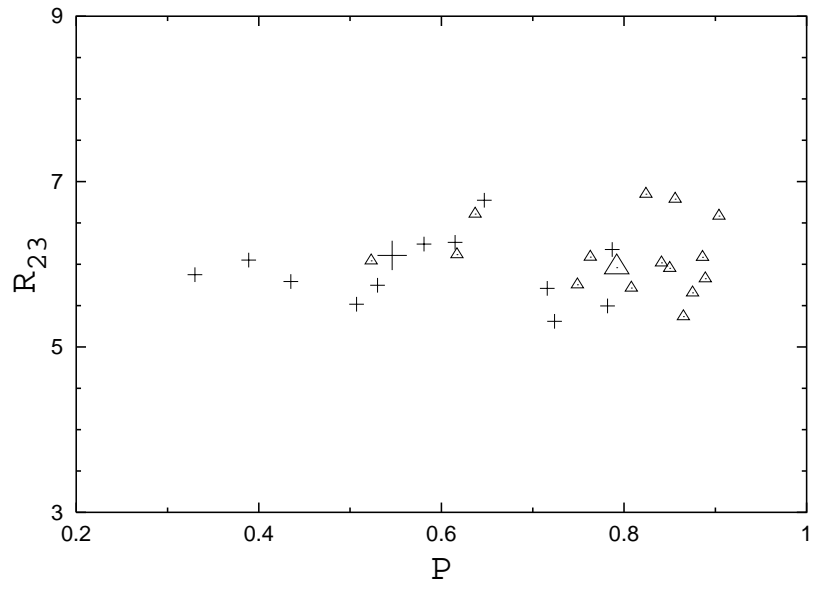

Fig. 1. The $P-R_{23}$ diagram. The small triangles are measurements for individual areas of the HII region M17 in the Milky Way Galaxy (Peimbert et al. 1992), the large triangle is the integrated data. The small pluses are measurements for individual areas of the HII region DEM323 in the Large Magellanic Cloud, the large plus is the integrated data (Oey et al. 2000)

intensities $-\mathrm{O} / \mathrm{H}$ calibration is derived in Sect. 3. The line intensities $-T_{\mathrm{e}}$ calibration is derived in Sect. 4 . A discussion is presented in Sect. 5. Section 6 contains a brief summary.

\section{Preliminary analysis of observational data}

The strategy for construction of an empirical relation between strong line intensities and oxygen abundance is based on the following propositions 1) the value of $\mathrm{O} / \mathrm{H}$ in high-metallicity HII region can be expressed as a function of two parameters: the value of $R_{23}$ and the hardness of the ionizing radiation, 2) the excitation index $P$ is a good indicator of the hardness of the ionizing radiation. (Notations similar to those of Paper I will be adopted here: $R_{2}=I_{[\mathrm{OII}] \lambda 3727+\lambda 3729} / I_{\mathrm{H} \beta}, R_{3}=I_{[\mathrm{OIII}] \lambda 4959+\lambda 5007} / I_{\mathrm{H} \beta}$, $R=I_{[\mathrm{OIII}] \lambda 4363} / I_{\mathrm{H} \beta}, R_{23}=R_{2}+R_{3}, X_{23}=\log R_{23}$, and $P=R_{3} / R_{23}$. The excitation index $P$ used here and indexes $p_{2}$ and $p_{3}$ used in Paper I are related through simple expressions: $p_{3}=\log P$ and $p_{2}=\log (1-P)$.) These propositions are immediately evident from the observational data. It has been shown that the value of $R_{23}$ is a robust property of a given HII region (Kennicutt et al. 2000; Oey et al. 2000) in the sense that its value is relatively constant within a given HII region. As an illustration of this fact, Fig. 1 shows the $R_{23}-P$ diagram for the multiple positions in the HII region M17 (Milky Way Galaxy) and in the HII region DEM323 (Large Magellanic Cloud). The M17 data include spectra from Peimbert et al. (1992) and the DEM323 data are from Oey et al. (2000). Both HII regions show a large range of excitation across the regions sampled; at the same time the value of $R_{23}$ is relatively constant. Other examples can be found in Kennicutt et al. (2000) and Oey et al. (2000).

On the other hand, Fig. 1 shows that the value of $R_{23}$ is not a good indicator of metal abundance in HII regions 
in the sense that there is no one-to-one correspondence between $R_{23}$ and oxygen abundance. Indeed, the HII regions M17 and DEM323 have in fact the same values of $R_{23}$, while their oxygen abundances are rather different: $12+\log \mathrm{O} / \mathrm{H}=8.37$ in DEM323 versus $12+\log \mathrm{O} / \mathrm{H}=8.61$ in M17. Thus the value of $R_{23}$ is governed not only by the oxygen abundance but at least one additional parameter. It is usually accepted that the spectrum of an HII region (and hence the value of $R_{23}$ ) is specified by three parameters: the abundance of the chemical elements in the gas, the ionization parameter or geometrical factor, and the hardness of the ionizing radiation (Stasinska 1990; McGaugh 1991, among others). The relative constancy of $R_{23}$ within a given nebula suggests that its value in moderately metal-rich HII regions depends very weakly (if at all) on the ionization parameter. This leads us to anticipate that the value of $\mathrm{O} / \mathrm{H}$ is a function of two parameters: the value of $R_{23}$ and the hardness of the ionizing radiation. Thus, an important proposition that in moderately high-metallicity HII regions $R_{23}$ is mainly governed by the oxygen abundance and by the hardness of the ionizing radiation (or effective temperature of the exciting star(s)) is immediately evident from the following observational facts: 1) the value of $R_{23}$ is relatively constant within a given HII region, 2) there is no one-to-one correspondence between $R_{23}$ and oxygen abundance.

It has been found that the value of $[\mathrm{OIII}] / \mathrm{H}_{\beta}$ correlates with the effective temperature of the exciting star or the hardness of the ionizing radiation (Kaler 1978; Copetti et al. 1986, among others) and hence the value of $[\mathrm{OIII}] / \mathrm{H}_{\beta}$ can be used as indicator of the hardness of the ionizing radiation. Vilchez \& Pagel (1988) have introduced the value

$\underline{[\mathrm{OII}] \lambda \lambda 3727,3729 /[\mathrm{OIII}] \lambda \lambda 4959,5007}$

$[\mathrm{SII}] \lambda \lambda 6716,6731 /[\mathrm{SIII}] \lambda \lambda 9069,9532$

as a hardness index. $[\mathrm{OIII}] / \mathrm{H}_{\beta}$ is not a very good indicator of the radiation hardness because it is affected by the oxygen abundance as well. The hardness index of Vilchez and Pagel contains the intensity of line [SIII] $\lambda$ 9532, which is often unknown. It will be assumed here that the excitation index $P$ can be used as indicator of the hardness of the ionizing radiation. The observational evidence in favor of this proposition is given in Fig. 2; the "calibrating" objects from Kennicutt et al. (2000) show a clearly defined correlation between the excitation index $P$ and effective temperature of the ionizing star.

The spectra of part but not the whole HII region are often observed. It should be noted that the value of $P$ is a good indicator of the hardness of the ionizing radiation only if measured fluxes reflect their relative contributions to the radiation of the whole nebula. This fact, together with the quality of spectra, has been taken into account in compiling the sample of HII regions used for the construction of an empirical relation between strong line intensities and oxygen abundance. If the measurements for individual areas in the same HII region were reported in a paper, the integrated spectra were derived (if authors did

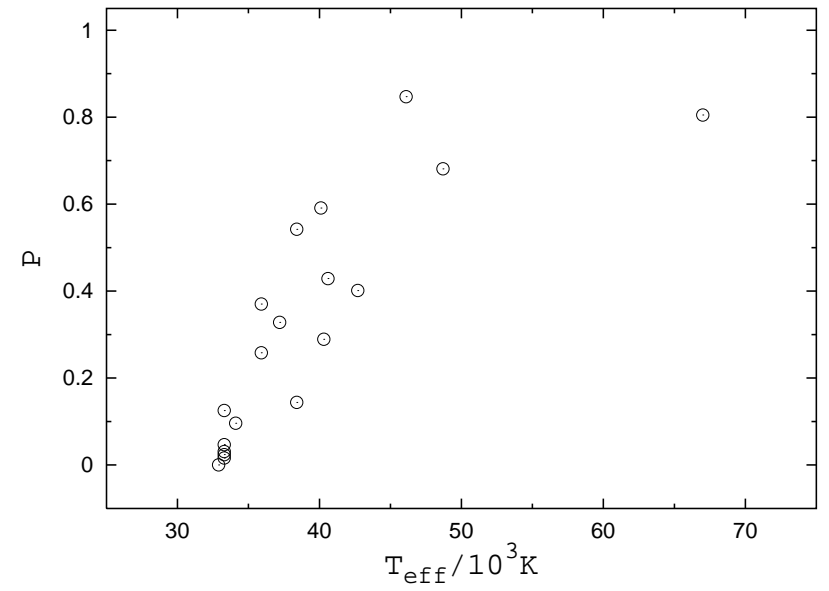

Fig. 2. The $P-T_{\text {eff }}$ diagram for the "calibrating" objects from Kennicutt et al. (2000)

not do so). If several spectra of equal quality were available for the same HII region, the spectrum with largest $\mathrm{H}_{\beta}$ flux was preferred. Our sample includes $38 \mathrm{HII}$ regions with $12+\log (\mathrm{O} / \mathrm{H})_{T_{\mathrm{e}}}>8.2$ for which we have collected the relevant observational data, listed with references in Table 1.

It is widely accepted that an accurate oxygen abundance can be derived from measurement of temperaturesensitive line ratios, such as $[\mathrm{OIII}] \lambda \lambda 4959,5007 / \lambda 4363$, i.e through the $T_{\mathrm{e}}$-method. In the general case, the oxygen abundances in the same HII region with measured line ratios $[\mathrm{OIII}] \lambda \lambda 4959,5007 / \lambda 4363$ derived in different works can differ for three reasons: atomic data adopted, interpretation of the temperature structure (single characteristic $T_{\mathrm{e}}$, two-zone model for $T_{\mathrm{e}}$, model with small-scale temperature fluctuations) and errors in the line intensity measurements. Therefore the compilation of HII regions with original oxygen abundance determinations through the $T_{\mathrm{e}}$ method from different works carried out over more than twenty years is not a set of homogeneous determinations. Accordingly, the available published spectra of HII regions with measured line ratios [OIII] $\lambda \lambda 4959,5007 / \lambda 4363$ (listed in the Table 1) have been reanalysed to produce a homogeneous set. Two-zone models of HII regions with the algorithm for oxygen abundance determination from Pagel et al. (1992) and $T_{\mathrm{e}}([\mathrm{OII}])-T_{\mathrm{e}}([\mathrm{OIII}])$ relation from Garnett (1992) were adopted here. The oxygen abundances for HII regions from Table 1 were recomputed in this common way. The obtained electron temperature $T_{\mathrm{e}}$ and corresponding oxygen abundance $\mathrm{O} / \mathrm{H}_{T_{\mathrm{e}}}$ are reported in Cols. 8 and 10 of Table 1 . The electron temperatures derived by Garnett et al. (1997) were used for NGC 2403 HII regions (the $R=I_{[\mathrm{OIII}] \lambda 4363} / I_{\mathrm{H} \beta}$ values for NGC 2403 HII regions reported in Table 1 correspond to these electron temperatures but not to the measurements). This set of HII regions with homogeneous determinations will be used in the construction of an empirical relation between strong line intensities and oxygen abundance. 
Table 1. Characteristics for the HII regions in the present sample. The commonly used name of the galaxy is given in Col. 1, the name of the HII region is reported in Col. 2. The fluxes $R_{2}=I_{[\mathrm{OII}] \lambda 3727+\lambda 3729} / I_{\mathrm{H} \beta}, R_{3}=I_{[\mathrm{OIII}] \lambda 4959+\lambda 5007} / I_{\mathrm{H} \beta}$, $R=I_{[\mathrm{OIII}] \lambda 4363} / I_{\mathrm{H} \beta}$ are listed in Cols. 3 to 5 . The electron concentration $N_{\mathrm{e}}$ is reported in Col. 6. The source for fluxes and electron concentration is listed in Col. 7. The electron temperature $T_{\mathrm{e}}$ derived from the measured $R_{3} / R$ line ratio is given in Col. 8. The electron temperature $t_{P}$ determined with the suggested $T_{\mathrm{e}}=f\left(P, R_{23}\right)$ relation is reported in Col. 9. The electron temperatures are given in units of $10^{4} \mathrm{~K}$. The oxygen abundance $\mathrm{O} / \mathrm{H}_{T_{\mathrm{e}}}$ derived through the $T_{\mathrm{e}}$-method with $T_{\mathrm{e}}$ is listed in Col. 10. The oxygen abundance $\mathrm{O} / \mathrm{H}_{P}$ determined via suggested $\mathrm{O} / \mathrm{H}=f\left(P, R_{23}\right)$ relation is given in Col. 11. The oxygen abundance $\mathrm{O} / \mathrm{H}_{t_{P}}$ derived through the $T_{\mathrm{e}}$-method with $t_{P}$ is listed in Col. 12. The oxygen abundances are given in units of $12+\log \mathrm{O} / \mathrm{H}$

\begin{tabular}{|c|c|c|c|c|c|c|c|c|c|c|c|}
\hline galaxy & HII region & $R_{2}$ & $R_{3}$ & $R$ & $N_{\mathrm{e}}$ & references & $T_{\mathrm{e}}$ & $t_{P}$ & $\overline{\mathrm{O} / \mathrm{H}_{T_{\mathrm{e}}}}$ & $\overline{\mathrm{O} / \mathrm{H}_{P}}$ & $\overline{\mathrm{O} / \mathrm{H}_{t_{P}}}$ \\
\hline \multirow[t]{9}{*}{ MWG } & $\mathrm{M} 16=\mathrm{Sh} 49$ & 1.429 & 1.262 & 0.0012 & 124 & $\mathrm{C}+\mathrm{D}$ & 0.66 & 0.63 & 8.58 & 8.68 & 8.67 \\
\hline & Sh117 & 1.799 & 1.506 & 0.0018 & 17 & $\mathrm{C}+\mathrm{D}$ & 0.69 & 0.69 & 8.59 & 8.60 & 8.60 \\
\hline & Sh184 & 1.900 & 1.762 & 0.0032 & 65 & $\mathrm{C}+\mathrm{D}$ & 0.76 & 0.72 & 8.48 & 8.58 & 8.58 \\
\hline & Sh206 & 1.189 & 4.637 & 0.0137 & 412 & $\mathrm{C}+\mathrm{D}$ & 0.85 & 0.80 & 8.47 & 8.57 & 8.58 \\
\hline & Sh212 & 3.102 & 1.741 & 0.0068 & 126 & $\mathrm{C}+\mathrm{D}$ & 0.92 & 0.88 & 8.32 & 8.38 & 8.38 \\
\hline & M42=Orion & 1.355 & 3.912 & 0.0101 & 3577 & $\mathrm{C}+\mathrm{D}$ & 0.82 & 0.78 & 8.55 & 8.59 & 8.64 \\
\hline & M17 & 1.239 & 4.720 & 0.010 & 691 & P92 & 0.79 & 0.81 & 8.61 & 8.56 & 8.57 \\
\hline & S298=RCW5-1 & 0.912 & 9.119 & 0.0708 & 200 & $\mathrm{~S} 83$ & 1.13 & 1.06 & 8.27 & 8.34 & 8.36 \\
\hline & $\mathrm{S} 311=\mathrm{RCW} 16-1$ & 2.344 & 3.861 & 0.0107 & 200 & $\mathrm{~S} 83$ & 0.84 & 0.90 & 8.54 & 8.42 & 8.43 \\
\hline \multirow[t]{2}{*}{ NGC 55} & No. 2 & 2.66 & 5.92 & 0.032 & 100 & W83 & 1.01 & 1.09 & 8.40 & 8.28 & 8.29 \\
\hline & No. 7 & 2.61 & 5.83 & 0.033 & 100 & W83 & 1.02 & 1.07 & 8.37 & 8.29 & 8.30 \\
\hline NGC 300 & No. 15 & 2.90 & 5.56 & 0.044 & 100 & W83 & 1.14 & 1.10 & 8.23 & 8.26 & 8.28 \\
\hline \multirow[t]{2}{*}{ NGC 598} & NGC 588 & 1.482 & 6.310 & 0.024 & 100 & V88 & 0.91 & 0.94 & 8.48 & 8.43 & 8.44 \\
\hline & NGC 604 & 2.152 & 2.852 & 0.0075 & 100 & D87 & 0.83 & 0.81 & 8.47 & 8.50 & 8.50 \\
\hline NGC 925 & $-022+227$ & 2.04 & 5.653 & 0.022 & 100 & vZ98 & 0.92 & 0.98 & 8.48 & 8.38 & 8.39 \\
\hline \multirow[t]{2}{*}{ NGC 1313} & No. 5 & 1.66 & 6.26 & 0.026 & 100 & P80 & 0.93 & 0.96 & 8.46 & 8.40 & 8.41 \\
\hline & No. 7 & 3.63 & 4.70 & 0.039 & 100 & P80 & 1.16 & 1.17 & 8.22 & 8.20 & 8.21 \\
\hline NGC 1569 & $\mathrm{C} 6 \mathrm{w}$ & 1.112 & 9.018 & 0.077 & 100 & K97 & 1.17 & 1.08 & 8.23 & 8.31 & 8.34 \\
\hline \multirow[t]{5}{*}{ NGC 2403} & VS 35 & 2.46 & 1.84 & 0.0051 & 100 & G97 & 0.84 & 0.79 & 8.40 & 8.48 & 8.48 \\
\hline & VS 38 & 1.92 & 1.34 & 0.0025 & 100 & G97 & 0.76 & 0.69 & 8.43 & 8.59 & 8.59 \\
\hline & VS 44 & 2.8 & 1.99 & 0.0063 & 100 & G97 & 0.87 & 0.85 & 8.39 & 8.42 & 8.42 \\
\hline & VS 51 & 2.28 & 2.37 & 0.0054 & 100 & G97 & 0.80 & 0.80 & 8.50 & 8.50 & 8.50 \\
\hline & VS 3 & 2.26 & 2.14 & 0.0068 & 100 & G97 & 0.87 & 0.79 & 8.35 & 8.51 & 8.51 \\
\hline \multirow[t]{3}{*}{ NGC 2805} & $+037-115$ & 2.52 & 4.662 & 0.020 & 100 & vZ98 & 0.94 & 0.98 & 8.42 & 8.36 & 8.37 \\
\hline & $-068-079$ & 3.64 & 4.552 & 0.033 & 100 & vZ98 & 1.11 & 1.16 & 8.26 & 8.21 & 8.22 \\
\hline & $+116-098$ & 3.11 & 4.681 & 0.030 & 100 & vZ98 & 1.07 & 1.07 & 8.29 & 8.27 & 8.28 \\
\hline \multirow[t]{2}{*}{ NGC 4214} & Knot2 (A6) & 3.172 & 3.746 & 0.021 & 100 & K96 & 1.02 & 1.02 & 8.31 & 8.31 & 8.31 \\
\hline & Knot5 (C6) & 2.557 & 5.371 & 0.024 & 100 & K96 & 0.96 & 1.03 & 8.44 & 8.32 & 8.33 \\
\hline NGC 4395 & $-272+186$ & 3.06 & 4.968 & 0.024 & 100 & vZ98 & 0.98 & 1.09 & 8.42 & 8.27 & 8.28 \\
\hline \multirow[t]{5}{*}{ NGC 5457} & NGC 5447 & 1.90 & 5.50 & 0.028 & 100 & $\mathrm{~S} 75$ & 0.99 & 0.95 & 8.35 & 8.41 & 8.42 \\
\hline & NGC 5455 & 3.09 & 5.25 & 0.028 & 100 & $\mathrm{~S} 75$ & 1.01 & 1.11 & 8.40 & 8.25 & 8.26 \\
\hline & NGC 5461 & 2.14 & 4.04 & 0.015 & 234 & T89 & 0.90 & 0.89 & 8.42 & 8.45 & 8.45 \\
\hline & $-347+276$ & 0.98 & 7.906 & 0.036 & 100 & vZ98 & 0.96 & 0.98 & 8.45 & 8.40 & 8.42 \\
\hline & $-459-053$ & 3.00 & 3.996 & 0.022 & 100 & vZ98 & 1.02 & 1.01 & 8.32 & 8.32 & 8.32 \\
\hline LMC & DEM323 & 2.770 & 3.337 & 0.014 & 10 & $\mathrm{O}+\mathrm{O}$ & 0.94 & 0.93 & 8.37 & 8.38 & 8.38 \\
\hline UM311 & & 1.800 & 5.295 & 0.023 & 100 & I98 & 0.95 & 0.92 & 8.40 & 8.44 & 8.44 \\
\hline UGC 2984 & No. 2 & 2.592 & 6.499 & 0.048 & 100 & vZ97 & 1.12 & 1.12 & 8.28 & 8.26 & 8.28 \\
\hline UGC 5716 & No. 1 & 2.640 & 5.025 & 0.031 & 100 & vZ97 & 1.05 & 1.02 & 8.30 & 8.32 & 8.33 \\
\hline
\end{tabular}

List of references :

C+D - Caplan et al. (2000) + Deharveng et al. (2000); D87 - Diaz et al. (1987); G97 - Garnett et al. (1997); I98 - Izotov \& Thuan (1998); K96 - Kobulnicky \& Skillman (1996); K97 - Kobulnicky \& Skillman (1997); O+O - Oey \& Shields (2000) + Oey et al. (2000); P80 - Pagel et al. (1980); P92 - Peimbert et al. (1992); S75 - Smith (1975); S83 - Shaver et al. (1983); T89 - Torres-Peimbert et al. (1989); V88 - Vilchez et al. (1988); vZ97 - van Zee et al. (1997); vZ98 - van Zee et al. (1998); W83 Webster \& Smith (1983). 


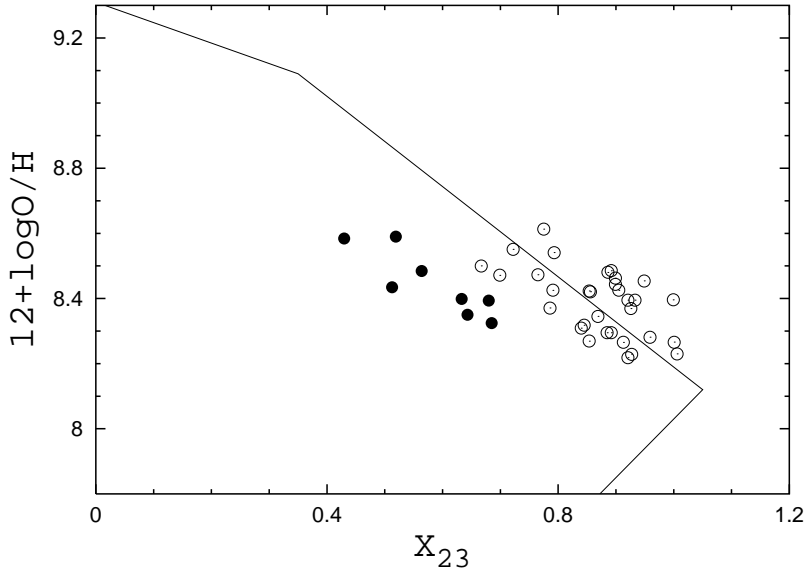

Fig. 3. The $X_{23}-\mathrm{O} / \mathrm{H}$ diagram for the HII regions from our sample. The filled circles are HII regions with $P<0.5$, open circles are HII regions with $P>0.5$. The line is the $R_{23}$ calibration after Edmunds and Pagel (1984)

\section{Line intensities $-\mathrm{O} / \mathrm{H}$ calibration}

The $X_{23}$ versus $\mathrm{O} / \mathrm{H}$ diagram for HII regions from Table 1 is presented in Fig. 3. The open circles are HII regions with $P>0.5$, the filled circles are HII regions with $P<0.5$. The line is the $R_{23}$ calibration of Edmunds and Pagel (1984). Inspection of Fig. 3 shows that there is no one-toone correspondence between $X_{23}$ value and oxygen abundance. For a fixed oxygen abundance the positions of lowexcitation $\mathrm{HII}$ regions in the $X_{23}-\mathrm{O} / \mathrm{H}$ diagram are systematically shifted towards lower values of $X_{23}$ compared to the positions of high-excitation HII regions. The calibration of Edmunds \& Pagel (1984) corresponds to the positions of high-excitation HII regions. This is not surprising since observational data only for high-excitation HII regions were available when this calibration was suggested. Other previous calibrations (McCall et al. 1985; Dopita \& Evans 1986; Zaritsky et al. 1994) are shifted towards still higher oxygen abundances. The fact that for a fixed oxygen abundance the value of $X_{23}$ varies with the excitation parameter $P$ confirms our proposition that the excitation index $P$ can be used in the oxygen abundance determination.

It is convenient to start the search for the relation between oxygen abundance and parameters $P$ and $R_{23}$ from consideration of $R_{3}-P$ (Fig. 4) and $R_{23}-P$ (Fig. 5) diagrams. The positions of HII regions from Table 1 in these diagrams are presented by circles; the filled circles are HII regions with $12+\log (\mathrm{O} / \mathrm{H})<8.4$, open circles are HII regions with $12+\log (\mathrm{O} / \mathrm{H})<8.4$. The points are HII regions from Zaritsky et al. (1994) and van Zee et al. (1998). The pluses are low-excitation HII regions in our Galaxy from Deharveng et al. (2000). Figure 4 shows that HII regions occupy rather narrow band in the $R_{3}-P$ diagram. The relation between $R_{3}$ and $P$ can be given by a polynomial

$k_{0} R_{3}=k_{1} P+k_{2} P^{2}+k_{3} P^{3}$.

The zero-degree term of the polynomial must be equal to zero since $R_{3}$ and $P$ are equal to zero simultaneously

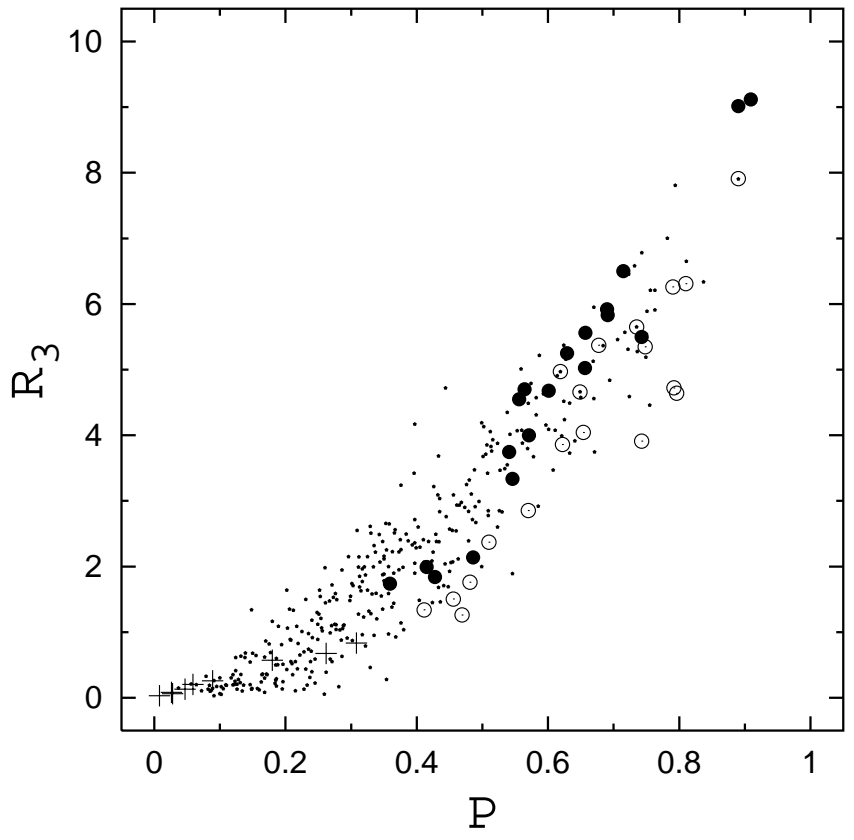

Fig. 4. The $P-R_{3}$ diagram. The HII regions from our sample are represented by circles; the filled circles are HII regions with $12+\log (\mathrm{O} / \mathrm{H})<8.4$, open circles are HII regions with $12+$ $\log (\mathrm{O} / \mathrm{H})>8.4$. The points are HII regions from Zaritsky et al. (1994) and van Zee et al. (1998). The pluses are low-excitation HII regions in our Galaxy from Caplan et al. (2000); Deharveng et al. (2000)

by the definition. Examination of Fig. 4 shows that the positions of the HII regions with $12+\log (\mathrm{O} / \mathrm{H})<8.4$ are shifted relative to those with $12+\log (\mathrm{O} / \mathrm{H})<8.4$. In order to take this fact into account the coefficients of Eq. (2) will be taken in the form

$k_{j}=a_{j}+b_{j} Z$,

where the notation $Z=12+\log \mathrm{O} / \mathrm{H}$ is used for brevity. Taking Eq. (3) into account, Eq. (2) can be rewritten as

$R_{3}=\frac{\left(a_{1}+b_{1} Z\right) P+\left(a_{2}+b_{2} Z\right) P^{2}+\left(a_{3}+b_{3} Z\right) P^{3}}{1+b_{0} Z}$.

The coefficient $a_{0}$ has been taken equal to 1 (deviding the numerator and denominator in the right side of Eq. (4) by $\left.a_{0}\right)$. Equation (4) can be solved for the value $Z=$ $12+\log \mathrm{O} / \mathrm{H}$

$12+\log (\mathrm{O} / \mathrm{H})_{P}=\frac{R_{3}-a_{1} P-a_{2} P^{2}-a_{3} P^{3}}{b_{1} P+b_{2} P^{2}+b_{3} P^{3}-b_{0} R_{3}}$.

Taking into account that $R_{3}=P \times R_{23}$, Eq. (5) can be transformed into

$12+\log (\mathrm{O} / \mathrm{H})_{P}=\frac{R_{23}-a_{1}-a_{2} P-a_{3} P^{2}}{b_{1}+b_{2} P+b_{3} P^{2}-b_{0} R_{23}}$.

The coefficients $b_{0}, a_{1}, b_{1}, a_{2}, b_{2}, a_{3}, b_{3}$ can be found using the set of HII regions with oxygen abundances derived through the $T_{\mathrm{e}}$-method. In other words, the positions in the $\mathrm{R}_{23}-P$ (and the $R_{3}-P$ ) diagram can be calibrated in terms of oxygen abundance. 


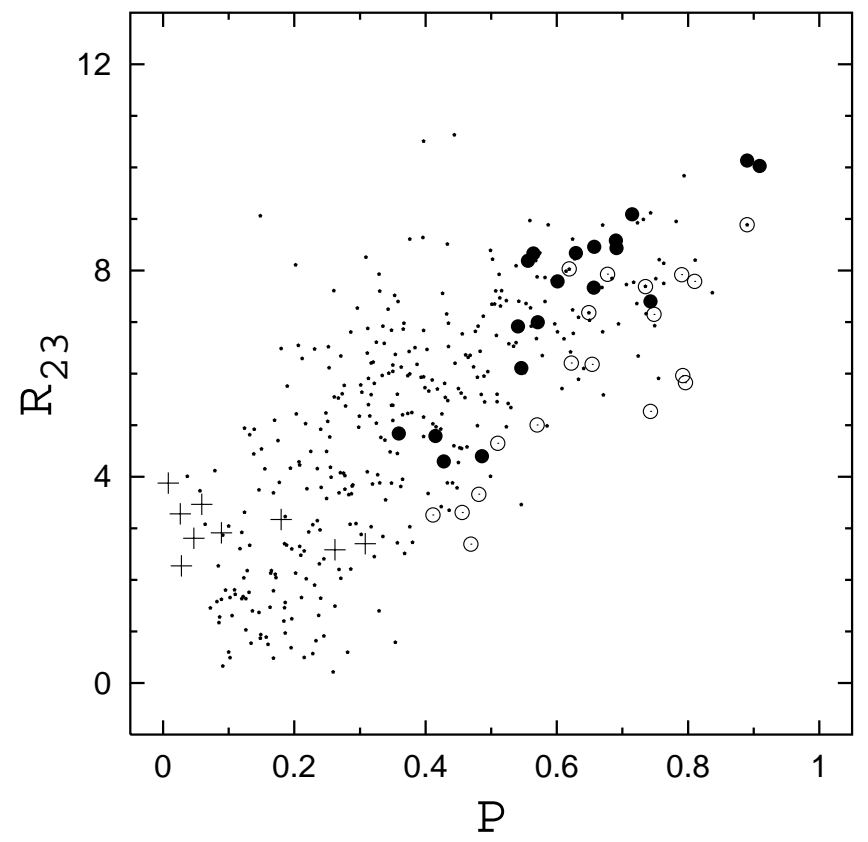

Fig. 5. The $P-R_{23}$ diagram. The HII regions from our sample are represented by circles; the filled circles are HII regions with $12+\log (\mathrm{O} / \mathrm{H})<8.4$, open circles are HII regions with $12+$ $\log (\mathrm{O} / \mathrm{H})>$ 8.4. The points are HII regions from Zaritsky et al. (1994) and van Zee et al. (1998). The pluses are low-excitation HII regions in our Galaxy from Caplan et al. (2000); Deharveng et al. (2000)

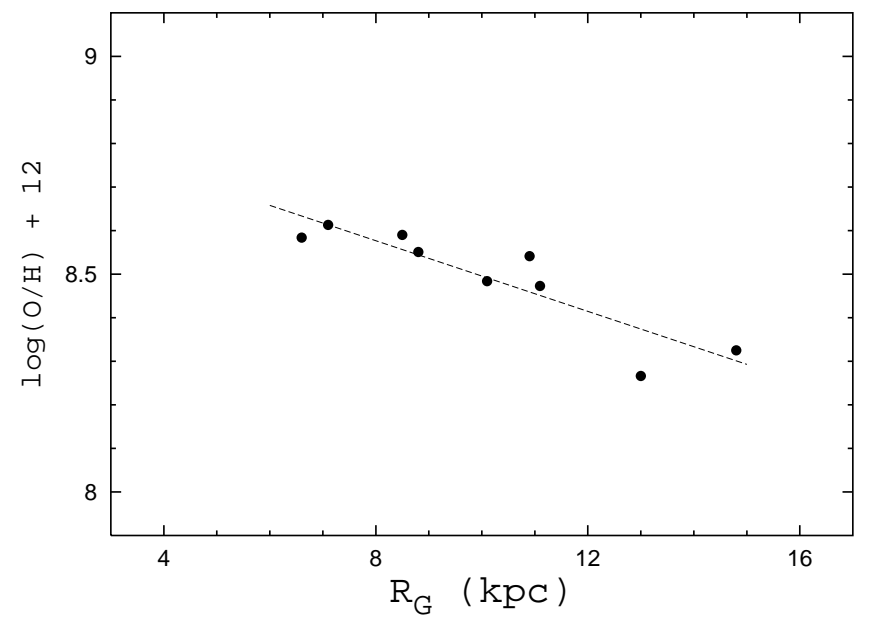

Fig. 6. The radial distribution of oxygen abundance in the disk of our Galaxy. The oxygen abundances of HII regions are determined through the $T_{\mathrm{e}}$-method (recomputed here); the distances are taken from Deharveng et al. (2000)

The precision of present-day determinations of the oxygen abundances in high-metallicity HII regions through the $T_{\mathrm{e}}$-method seems to be around $0.1 \mathrm{dex}$ (Deharveng et al. 2000). It has been found that there are different sets of coefficients $b_{0}, a_{1}, b_{1}, a_{2}, b_{2}, a_{3}, b_{3}$ (or different variants of the calibration) which give an average value of differences $\Delta \mathrm{O} / \mathrm{H}_{P}=\log \mathrm{O} / \mathrm{H}_{P}-\log \mathrm{O} / \mathrm{H}_{T_{\mathrm{e}}}$ less than 0.1 dex for our sample of HII regions. Unfortunately, our sample of HII regions (used in the search for the coefficients) does not contain the HII regions with $P<0.4$,

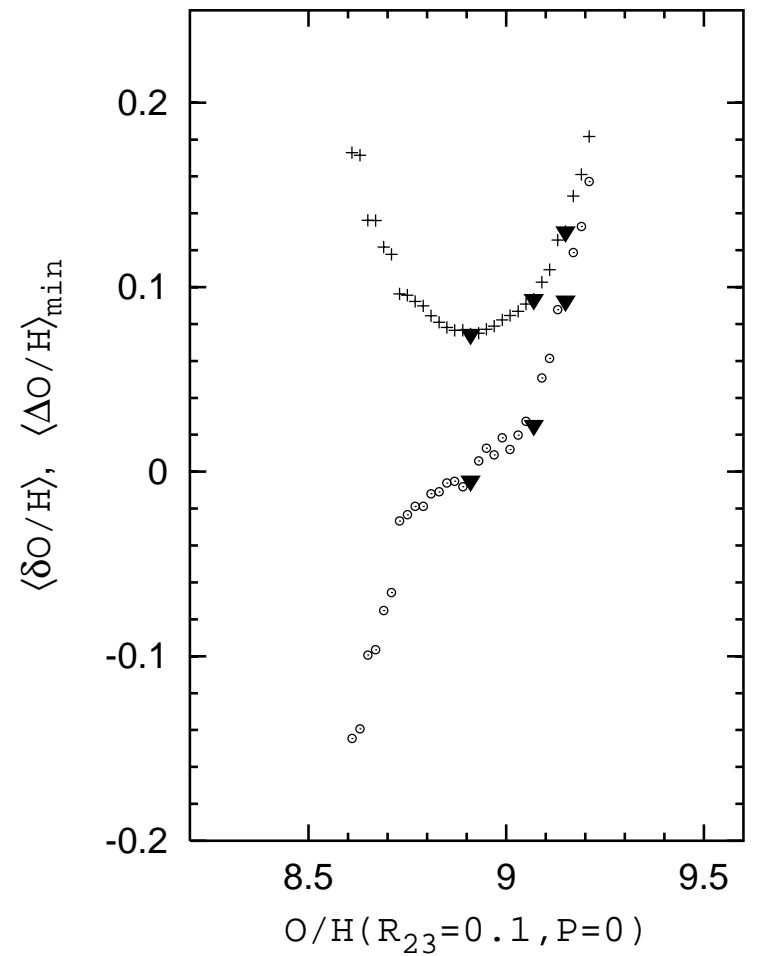

Fig. 7. The $\langle\Delta \mathrm{O} / \mathrm{H}\rangle_{\min }$ and $\langle\delta \mathrm{O} / \mathrm{H}\rangle$ as a function of the $\mathrm{O} / \mathrm{H}^{*}=\mathrm{O} / \mathrm{H}\left(R_{23}=0.1, P=0\right)$. The $\langle\Delta \mathrm{O} / \mathrm{H}\rangle_{\text {min }}$ values are presented by pluses, the $\langle\delta \mathrm{O} / \mathrm{H}\rangle$ values are presented by the circles. The variants of the calibration (V891, V907, and V915) discussed in the text are shown by filled triangles

Fig. 5. Therefore different sets of coefficients based on the narrow range of oxygen abundances in high-excitation HII regions can result in appreciably different abundances in low-excitation ones although all the variants of the calibration that resulted in a correlation between the differences $\Delta \mathrm{O} / \mathrm{H}_{j}$ for individual $\mathrm{HII}$ regions and $P_{j}$ or between $\Delta \mathrm{O} / \mathrm{H}_{j}$ and $\mathrm{O} / \mathrm{H}_{j}$ were rejected and only the variants in which both correlation coefficients are less than 0.1 were considered. Thus, the problem of choice of the variant of the calibration, which results in correct oxygen abundances in the whole range of $P$, appears. This difficulty is usually resolved by adding HII region models to the real HII regions. As was discussed by Stasinska (2000), the existing models of HII regions may be far from reality for a number of reasons. Then we may have to try to overcome this difficulty starting from the observational data only. The following solution to this problem will be adopted. Our sample contains 9 HII regions of the disk of our Galaxy. Figure 6 shows the oxygen abundance as a function of the galactocentric distance. The linear fit

$12+\log (\mathrm{O} / \mathrm{H})=8.90-0.041 R_{\mathrm{G}}$

is close to the relation obtained by Deharveng et al. (2000). This is not surprising, since the measured fluxes for 6 out of the 9 HII regions were taken from them. The list of HII regions observed by Caplan et al. (2000) and Deharveng et al. (2000) contains a number of low-excitation ones. Following Deharveng et al. (2000) all the heavily reddened 

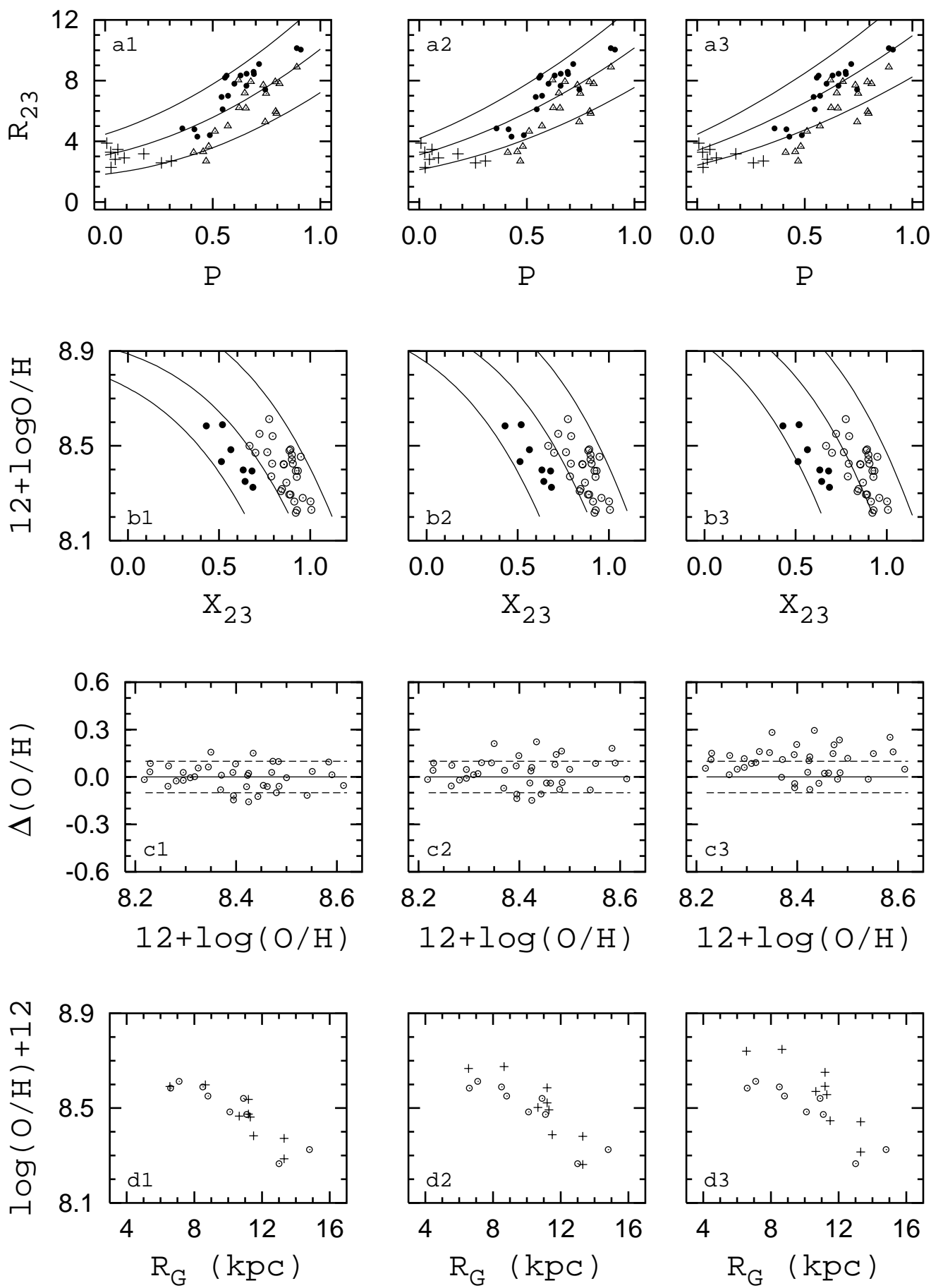

Fig. 8. The comparison of three variants of the calibration (V891 - 1st column panels, V907 - 2nd column panels, V915 3rd column panels) with observational data. The panels a1, a2, and a3 show the $R_{23}-P$ diagrams. The filled circles are HII regions with $8.2<12+\log \mathrm{O} / \mathrm{H}<8.4$, the open triangles are $\mathrm{HII}$ regions with $8.4<12+\log \mathrm{O} / \mathrm{H}<8.62$ from our sample. The pluses are low-excitation HII regions in the disk of our Galaxy from Deharveng et al. (2000). The curves are $R_{23}-P$ relations predicted by a given variant of the calibration for fixed values of $\log \mathrm{O} / \mathrm{H}+12=8.2, \log \mathrm{O} / \mathrm{H}+12=8.4, \log \mathrm{O} / \mathrm{H}+12=$ 8.6 (from top to bottom). The panels b1, b2, and b3 show the $X_{23}-\mathrm{O} / \mathrm{H}$ diagrams. The filled circles are HII regions with $P<0.5$, the open circles are HII regions with $P>0.5$ from our sample. The curves are $X_{23}-\mathrm{O} / \mathrm{H}$ relations predicted by a given variant of the calibration for fixed values of $P=1, P=0.5$, and $P=0$ (from top to bottom). The panels c1, c2, and c3 show $\Delta \mathrm{O} / \mathrm{H}=\log (\mathrm{O} / \mathrm{H})_{P}-\log (\mathrm{O} / \mathrm{H})_{T_{\mathrm{e}}}$ versus $\mathrm{O} / \mathrm{H}_{T_{\mathrm{e}}}$ diagrams for our sample of HII regions. The panels d1, d2, and d3 show the radial distribution of oxygen abundance within the disk of our Galaxy. The circles are HII regions with oxygen abundances derived through the $T_{\mathrm{e}}$-method, the pluses are low-excitation HII regions with oxygen abundances determined through the given variant of the present calibration 
HII regions were excluded from consideration. The nine low-excitation HII regions Sh54, Sh131, Sh148, Sh 152, Sh153, Sh156, Sh168, Sh217, and Sh219 for which flux measurements seems to be reliable were selected and used as "secondary calibrating objects" in the choice of the relation between strong line intensities and oxygen abundance (the positions of these HII regions in the $R_{3}-P$ and $R_{23}-P$ diagrams are shown by pluses, Figs. 4 and $5)$. The basic requirement imposed on the calibration by our set of HII regions is complemented by the condition that the oxygen abundances of selected low-excitation HII regions derived through the calibration must result in the same radial oxygen abundance gradient as was determined from HII regions with oxygen abundances derived through the $T_{\mathrm{e}}$-method. Inspection of Fig. 5 shows that positions of HII regions from this "extended" set cover the whole range of $P$.

The choice of the variant of the calibration which is suitable for the whole range of the parameter $P$ was performed in the following way. A variant of the calibration can be characterized by three parameters; $\mathrm{O} / \mathrm{H}^{*}=$ $\mathrm{O} / \mathrm{H}\left(R_{23}=0.1, P=0\right),\langle\Delta \mathrm{O} / \mathrm{H}\rangle=\sqrt{\left(\sum_{j=1}^{n}\left(\Delta \mathrm{O} / \mathrm{H}_{j}\right)^{2}\right) / n}$, and $\langle\delta \mathrm{O} / \mathrm{H}\rangle=\left(\sum_{j=1}^{n} \Delta \mathrm{O} / \mathrm{H}_{j}\right) / n$ where $\Delta \mathrm{O} / \mathrm{H}_{j}$ is equal to $\log \mathrm{O} / \mathrm{H}_{P}^{j}-\log \mathrm{O} / \mathrm{H}_{T_{\mathrm{e}}}^{j}$ for our set of HII regions and $\Delta \mathrm{O} / \mathrm{H}_{j}$ is equal to $\log \mathrm{O} / \mathrm{H}_{P}^{j}-\log \mathrm{O} / \mathrm{H}_{R_{\mathrm{G}}}^{j}$ for the selected low-excitation HII regions where $\mathrm{O} / \mathrm{H}_{R_{\mathrm{G}}}^{j}$ is the abundance estimated through the galactocentric distance (Eq. (7)). The $\mathrm{O} / \mathrm{H}^{*}$ is the minimum value of oxygen abundance for HII regions with $R_{23}=0.1$, predicted by a given variant of the calibration. The value of $\langle\Delta \mathrm{O} / \mathrm{H}\rangle$ characterizes the scatter of differences between oxygen abundances determined via a fixed variant of the calibration and adopted oxygen abundances. The value of $\langle\delta \mathrm{O} / \mathrm{H}\rangle$ characterizes the average systematic difference between oxygen abundances determined via the fixed variant of the calibration and adopted oxygen abundances. For every fixed value of the parameter $\mathrm{O} / \mathrm{H}^{*}$ the variant of the calibration which gives the minimum value of $\langle\Delta \mathrm{O} / \mathrm{H}\rangle$ was obtained. The value of $\langle\Delta \mathrm{O} / \mathrm{H}\rangle_{\min }$ and corresponding value of $\langle\delta \mathrm{O} / \mathrm{H}\rangle$ as a function of the $\mathrm{O} / \mathrm{H}^{*}$ are presented in Fig. 7. The local nonsmooth variations of the values of $\langle\Delta \mathrm{O} / \mathrm{H}\rangle_{\min }$ and $\langle\delta \mathrm{O} / \mathrm{H}\rangle$ with $\mathrm{O} / \mathrm{H}^{*}$ reflect the fact that all the variants of the calibration which result in a correlation between $\Delta \mathrm{O} / \mathrm{H}_{j}$ and $P_{j}$ or between $\Delta \mathrm{O} / \mathrm{H}_{j}$ and $\mathrm{O} / \mathrm{H}_{j}$ were rejected and only the variants in which both correlation coefficients are less than 0.1 were considered.

Figure 7 shows that the $\langle\Delta \mathrm{O} / \mathrm{H}\rangle_{\min }$ has a minimum value for $\mathrm{O} / \mathrm{H}^{*}=8.91$. The corresponding value of $\langle\delta \mathrm{O} / \mathrm{H}\rangle$ is close to zero $(\approx-0.005 \mathrm{dex})$. This variant of the calibration will be referred to as V891. The comparison of this variant of the calibration with observational data is given in Fig. 8 (1st column). Two other variants V907 and V915 of the calibration are also presented in Fig. 8 (panels in Cols. 2 and 3). Figure 8 shows that the V891 variant of the calibration well reproduces the observational data of different types. The positions of HII galaxies from our sample in the $R_{23}-P$ and $\mathrm{O} / \mathrm{H}-R_{23}$ diagrams are well reproduced by the V891 variant of the calibration (panels a1 and b1 in Fig. 8). The differences between oxygen abundances determined through the variant V891 of the calibration and through the $T_{\mathrm{e}}$-method do not exceed 0.1 dex (the average difference is about 0.08 dex for 38 HII regions) for the majority of our set (panel c1 in Fig. 8). For comparison, the average difference between variant V915 and the $T_{\mathrm{e}}$-method is in excess of $0.1 \mathrm{dex}(\approx 0.13 \mathrm{dex})$ (panel c3 in Fig. 8). Consequently, variant V891 of the calibration results in the radial oxygen abundance gradient which is very close to that derived from HII regions with oxygen abundances determined through the $T_{\mathrm{e}}$-method (panel d1 in Fig. 8). Then, the variant V891 of the calibration is the most credible relation between strong line intensities and oxygen abundance. This variant of the calibration with coefficients $b_{0}=-0.243, a_{1}=-54.2, b_{1}=6.07$, $a_{2}=-59.45, b_{2}=6.71, a_{3}=-7.31, b_{3}=0.371$ and corresponding equation

$12+\log (\mathrm{O} / \mathrm{H})_{P}=\frac{R_{23}+54.2+59.45 P+7.31 P^{2}}{6.07+6.71 P+0.371 P^{2}+0.243 R_{23}}$

can be adopted for oxygen abundance determinations in moderately high-metallicity HII regions with undetectable temperature-sensitive line ratios. The oxygen abundances in HII regions from our sample determined with this calibration are given in Table 1 (Col. 11).

\section{Line intensities $-T_{\mathrm{e}}$ calibration}

The oxygen abundances $\mathrm{O} / \mathrm{H}_{P}$ derived in the previous section through the excitation parameter $P$ and abundance index $R_{23}$ are in agreement with those derived through the classical $T_{\mathrm{e}}$-method. This justifies the use of relations of the type $\mathrm{O} / \mathrm{H}=f\left(P, R_{23}\right)$ for oxygen abundance determination in high-metallicity HII regions with the undetectable [OIII]4363 line. It also provides evidence that the propositions which are at the basis of this relation $(i)$ that the value of abundance index is mainly governed by the oxygen abundance and by the hardness of the ionizing radiation and depends very weakly (if at all) on the ionization parameter, $i i)$ that the parameter $P$ can be used as indicator of the hardness of the ionizing radiation) seem to be close to reality. Conversely, if the physical conditions in high-metallicity nebulae are governed mainly by the oxygen abundance and by the hardness of the ionizing radiation and if observational values of $P$ and $R_{23}$ reflect these two, then it can be expected that the physical conditions in a nebula can be derived with help of observational values $P$ and $R_{23}$. The physical conditions in a nebula are reflected in the electron temperature, so that a relation of the type $T_{\mathrm{e}}=f\left(P, R_{23}\right)$ can be expected.

Figure 9 shows the positions of HII regions from Table 1 in the $R_{23}-P$ diagram. The HII regions with $T_{\mathrm{e}}<0.8$ are presented by circles, those with $0.8<T_{\mathrm{e}}<$ 1.0 are shown by pluses and those with $T_{\mathrm{e}}>1.0$ are shown 


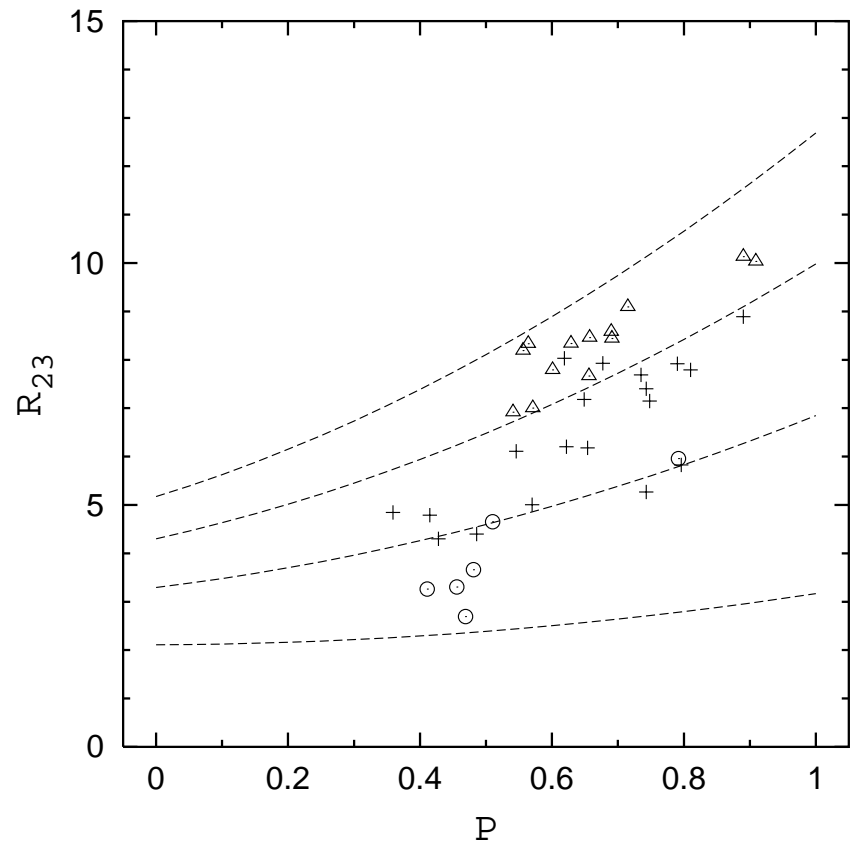

Fig. 9. The $P-R_{23}$ diagram. The HII regions with $T_{\mathrm{e}}<0.8$ are presented by circles, those with $0.8<T_{\mathrm{e}}<1.0$ are shown by pluses and those with $T_{\mathrm{e}}>1.0$ are shown by triangles. The curves are the $R_{23}-P$ relations predicted by the present calibration for a fixed values of $T_{\mathrm{e}}=0.6,0.8,1.0$, and 1.2 (from bottom to top)

by triangles. Figure 9 shows that the HII regions with different electron temperatures occupy different bands in the $R_{23}-P$ diagram. This suggests that the positions in the $R_{23}-P$ diagram can be calibrated in terms of electron temperatures. Let us again start from the expression of the type

$R_{3}=\frac{\left(A_{1}+B_{1} t\right) P+\left(A_{2}+B_{2} t\right) P^{2}+\left(A_{3}+B_{3} t\right) P^{3}}{1+B_{0} t}$,

which can be solved for the value of $t$

$t_{P}=\frac{R_{3}-A_{1} P-A_{2} P^{2}-A_{3} P^{3}}{B_{1} P+B_{2} P^{2}+B_{3} P^{3}-B_{0} R_{3}}$,

where $t_{P}$ is the electron temperature expressed in units of $10^{4} \mathrm{~K}$. The coefficients $B_{0}, A_{1}, B_{1}, A_{2}, B_{2}, A_{3}$, and $B_{3}$ can be found using the sample of HII regions with electron temperature derived via measured temperature-sensitive line ratios $[\mathrm{OIII}] 4959,5007 /[\mathrm{OIII}] 4363$. It has been found that there are different sets of coefficients $B_{0}, A_{1}, B_{1}$, $A_{2}, B_{2}, A_{3}$, and $B_{3}$ which provide the average value of differences $\Delta t_{P}=t_{P}-T_{\mathrm{e}}$ around $500 \mathrm{~K}$. The variant of the calibration (the choice is explained below) with coefficients $B_{0}=0.583, A_{1}=-3.09, B_{1}=9.90, A_{2}=-7.05, B_{2}=$ 11.86, $A_{3}=-2.87, B_{3}=7.05$ and corresponding equation

$t_{P}=\frac{R_{23}+3.09+7.05 P+2.87 P^{2}}{9.90+11.86 P+7.05 P^{2}-0.583 R_{23}}$

has been adopted for the electron temperature determination in high-metallicity HII regions with undetectable

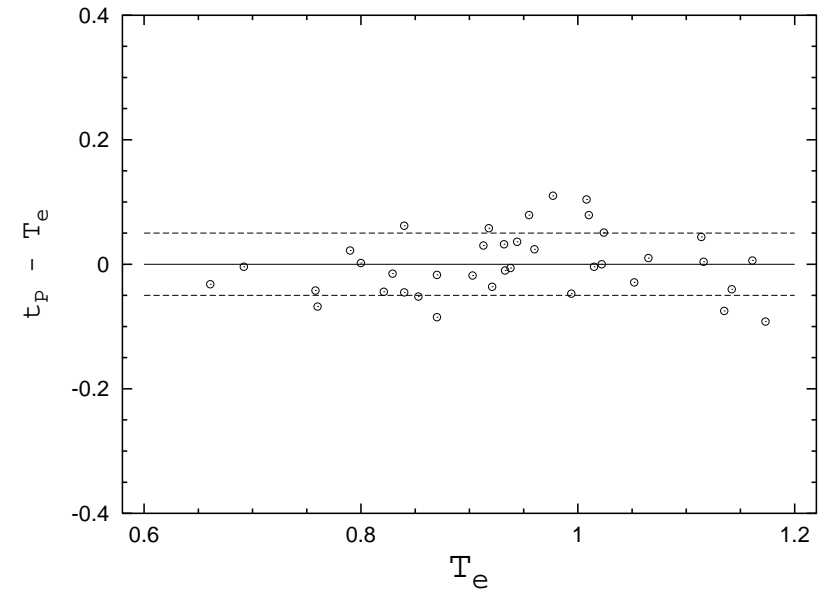

Fig. 10. The differences $t_{P}-T_{\mathrm{e}}$ as a function of $T_{\mathrm{e}}$, for our set of HII regions

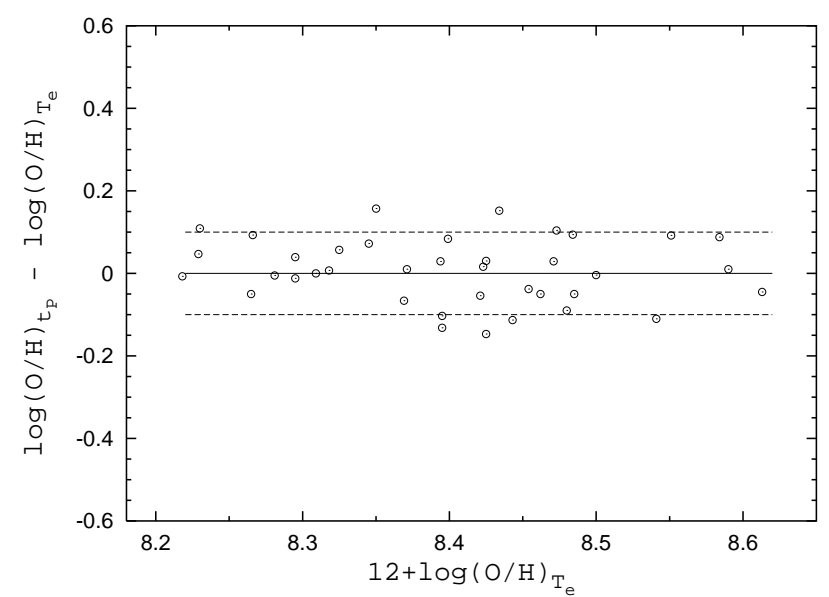

Fig. 11. The differences $(\mathrm{O} / \mathrm{H})_{t_{P}}-(\mathrm{O} / \mathrm{H})_{T_{\mathrm{e}}}$ for our set of HII regions

temperature-sensitive line ratios. The electron temperatures $t_{P}$ in HII regions from our sample determined from Eq. (11) are given in Table 1 (Col. 9).

The $R_{23}-P$ relations predicted by the calibration for fixed values of $t_{P}=0.6,0.8,1.0$, and 1.2 are presented in Fig. 9 by dashed lines. Figure 10 shows the differences between electron temperatures $t_{P}$ derived through the present calibration and measured electron temperatures $T_{\mathrm{e}}$. As can be seen in Fig. 10, the largest value of the difference $\Delta T_{\mathrm{e}}=t_{P}-T_{\mathrm{e}}$ is around $1000 \mathrm{~K}$, the average value about $500 \mathrm{~K}$.

Since the electron temperatures $t_{P}$ derived through the present calibration are close to the measured electron temperatures $T_{\mathrm{e}}$, the $t_{P}$ can be used for the oxygen abundance determination through the $T_{\mathrm{e}}$-method. The oxygen abundances $\mathrm{O} / \mathrm{H}_{t_{P}}$ in $\mathrm{HII}$ regions from our sample determined in this way are given in Table 1 (Col. 12). The differences between oxygen abundances derived with the $t_{P}$ and with the measured $T_{\mathrm{e}}$ are presented in Fig. 11. The variant of the $t_{P}=f\left(P, R_{23}\right)$ calibration which results in the best agreement between $\mathrm{O} / \mathrm{H}_{t_{P}}$ and $\mathrm{O} / \mathrm{H}_{T_{\mathrm{e}}}$ for our set of 


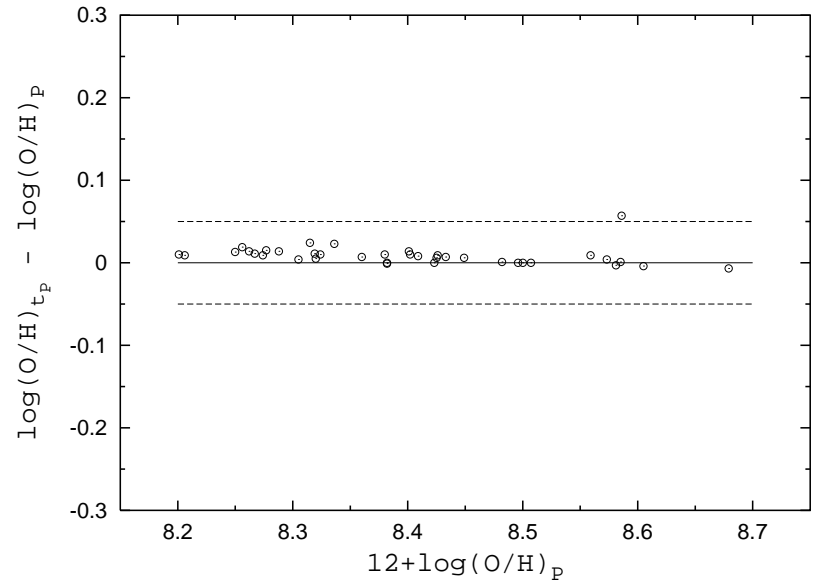

Fig. 12. The differences $(\mathrm{O} / \mathrm{H})_{t_{P}}-(\mathrm{O} / \mathrm{H})_{P}$ for our set of HII regions

HII regions and between $\mathrm{O} / \mathrm{H}_{t_{P}}$ and $\mathrm{O} / \mathrm{H}_{R_{\mathrm{G}}}$ for lowexcitation HII regions in the disk of our Galaxy was chosen above.

The differences between $\mathrm{O} / \mathrm{H}_{t_{P}}$ and $\mathrm{O} / \mathrm{H}_{P}$ for our set of HII regions are presented in Fig. 12. It can be seen in Fig. 12 that $\mathrm{O} / \mathrm{H}=f\left(P, R_{23}\right)$ and $t_{P}=f\left(P, R_{23}\right)$ relations lead to consistent abundances.

Thus, the positions in the $R_{23}-P$ diagram can be calibrated in terms of electron temperatures. The obtained relation $t_{P}=f\left(P, R_{23}\right)$ between electron temperature and parameters $P$ and $R_{23}$ provides an alternative (to the relation $\mathrm{O} / \mathrm{H}=f\left(P, R_{23}\right)$ obtained in the previous section) method of the oxygen abundance determination in highmetallicity HII regions with the undetectable [OIII]4363 line.

\section{Discussion}

The starting expression (Eq. (2)) for the search for the relation $T_{\mathrm{e}}=f\left(P, R_{23}\right)$ has been chosen from analysis of positions of HII regions in the $R_{3}-P$ (Fig. 4) and $R_{23}-P$ (Fig. 5) diagrams. These diagrams are based on directly measured values. The starting Eq. (2) can be rewritten in the form

$k_{0} R_{23}=k_{1}+k_{2} P+k_{3} P^{2}$,

where coefficients $\mathrm{k}_{j}$ are dependent on the electron temperature. With our assumption that the parameter $P$ is an indicator of the hardness of the ionizing radiation, Eq. (12) is the relation between the hardness of the ionizing radiation and electron temperature in the nebula. On the other hand, it is well known that the relation between the hardness of the ionizing radiation (or effective temperature of the exciting star) and electron temperature in the nebula can be derived from the law of energy conservation for free electrons. According to Sobolev (1967, Eq. (23.37)) this relation is given by the expression

$A T_{\mathrm{eff}}=B T_{\mathrm{e}}+C R_{23}+D\left\langle\frac{n_{1}}{n^{+}}\right\rangle$ where

$$
\left\langle\frac{n_{1}}{n^{+}}\right\rangle=\frac{\int n_{1} n_{\mathrm{e}} \mathrm{d} V}{\int n^{+} n_{\mathrm{e}} \mathrm{d} V},
$$

where coefficient $A$ depends on $T_{\text {eff }}$ only and $B, C$ and $D$ depend on $T_{\mathrm{e}}$ only. Comparison of Eq. (13) with Eq. (12) shows that with our assumption that the parameter $P$ is an indicator of the hardness of the ionizing radiation (or effective temperature of the exciting star) our starting Eq. (12) is in some sense similar to Eq. (13). Then, the relation $T_{\mathrm{e}}=f\left(P, R_{23}\right)$ derived here can be considered as an analog of the equation of the energy balance of a gaseous nebula.

The validity of the obtained relation $\mathrm{O} / \mathrm{H}=f\left(P, R_{23}\right)$ depends on the reliability of the oxygen abundances in HII regions for which this relation has been derived. The two-zone model for $T_{\mathrm{e}}$ is at the basis of the oxygen abundance determination in the present study. Then the $\mathrm{O} / \mathrm{H}=f\left(P, R_{23}\right)$ relation cannot be more credible than the two-zone model for $T_{\mathrm{e}}$. If it is ever established that the two-zone model for $T_{\mathrm{e}}$ is a crude approximation of reality and leads to large uncertainty in the oxygen abundances then the oxygen abundances in HII regions should be redetermined within the framework of a more realistic model and $\mathrm{O} / \mathrm{H}=f\left(P, R_{23}\right)$ relation should be revised.

Most metal-rich HII regions are objects with small values of $R_{23}$. Although both the $\mathrm{O} / \mathrm{H}_{P}$ and the $\mathrm{O} / \mathrm{H}_{R_{23}}$ are maximum for those $\mathrm{HII}$ regions, the $\mathrm{O} / \mathrm{H}_{P}$ for them are significantly lower (up to $0.5 \mathrm{dex}$ ) as compared to the $\mathrm{O} / \mathrm{H}_{R_{23}}$. It must be emphasized, however, that the $\mathrm{O} / \mathrm{H}=f\left(P, R_{23}\right)$ relation is established on the basis of HII regions with $R_{23}$ larger than 2 (panel a1 in Fig. 8) and the validity of this relation in the case of HII regions with $R_{23}$ less than 2 may be disputed. Then an additional information should be invoked in order to firmly establish how rich the most oxygen-rich HII regions are.

\section{Conclusions}

The problem of line intensity - oxygen abundance calibration has been investigated starting from the idea of McGaugh (1991) that the strong oxygen lines ([OII] $\lambda \lambda 3727,3729$ and $[\mathrm{OIII}] \lambda \lambda 4959,5007)$ contain the necessary information to determine accurate abundances in HII regions. The high-metallicity HII regions $(12+$ $\log \mathrm{O} / \mathrm{H} \geq 8.2$, the upper branch of the $\mathrm{O} / \mathrm{H}-R_{23}$ diagram) are considered in the present study. The lowmetallicity HII regions $(12+\log \mathrm{O} / \mathrm{H} \leq 7.95$, the lower branch of the $\mathrm{O} / \mathrm{H}-R_{23}$ diagram) have been considered in the previous study (Pilyugin 2000).

An important proposition that in high-metallicity HII regions the value of the abundance index $R_{23}$ is mainly governed by the oxygen abundance and by the hardness of the ionizing radiation (or by effective temperature of the exciting star(s)) and depends very weakly (if at all) on the ionization parameter, is immediately evident from the following observational facts: 1 ) the value of $R_{23}$ is relatively 
constant within a given HII region, 2) there is no one-toone correspondence between $R_{23}$ and oxygen abundance. Another important fact supported the use of the excitation parameter $P$ as an indicator of the hardness of the ionizing radiation is also evident from the observational data. These propositions are at the basis of the strong oxygen line intensity - oxygen abundance calibration.

A relation of the type $\mathrm{O} / \mathrm{H}=f\left(P, R_{23}\right)$ was derived empirically using the available oxygen abundances determined via measurement of temperature-sensivite line ratios $\left(T_{\mathrm{e}}\right.$-method). By comparing oxygen abundances in HII regions derived with the $T_{\mathrm{e}}$-method and those derived with the suggested relations $(P$-method) it was found that the precision of oxygen abundance determination with the $P$ method is around $0.1 \mathrm{dex}$ and is comparable to that obtained with the $T_{\mathrm{e}}$-method.

A relation of the type $T_{\mathrm{e}}=f\left(P, R_{23}\right)$ was derived empirically using the available electron temperatures determined via measurement of the temperature-sensivite line ratio $[\mathrm{OIII}] 4959,5007 /[\mathrm{OIII}] 4363$. The maximum value of differences between electron temperatures derived through the $T_{\mathrm{e}}=f\left(P, R_{23}\right)$ relation and determined via measurement of the temperature-sensitive line ratio is around $1000 \mathrm{~K}$ for HII regions considered here; the mean difference is $\sim 500 \mathrm{~K}$, which is of the same order of magnitude as the uncertainties of electron temperature determinations in high-metallicity HII regions via measured temperaturesensivite line ratios themselves.

The relation $T_{\mathrm{e}}=f\left(P, R_{23}\right)$ between electron temperature and parameters $P$ and $R_{23}$ provides an alternative (to the relation $\mathrm{O} / \mathrm{H}=f\left(P, R_{23}\right)$ ) method of oxygen abundance determination in high-metallicity HII regions with the undetectable [OIII]4363 line. By comparing oxygen abundances in HII regions derived through the $T_{\mathrm{e}^{-}}$ method with measured electron temperatures and those derived through the $T_{\mathrm{e}}$-method with electron temperature determined with help of $T_{\mathrm{e}}=f\left(P, R_{23}\right)$ relation, it was found that the precision of oxygen abundance determination with the latter method is around 0.1 dex and is comparable to that obtained with the $T_{\mathrm{e}}$-method.

Acknowledgements. I thank the referee, Prof. B. E. J. Pagel, for helpful comments and suggestions as well as improving the English text. This study was partly supported by the NATO grant PST.CLG.976036 and the Joint Research Project between Eastern Europe and Switzerland (SCOPE) No. 7 UKPJ62178.

\section{References}

Alloin, D., Collin-Souffrin, S., Joly, M., \& Vigrough, L. 1979, A\&A, 78, 200

Caplan, J., Deharveng, L., Pena, M., Costero, R., \& Blondel, C. 2000, MNRAS, 311, 317

Copetti, M. V., Pastoriza, M. G., \& Dottori, H. A. 1986, A\&A, 156,111

Deharveng, L., Pena, M., Caplan, J., \& Costero, R. 2000, MNRAS, 311, 329
Diaz, A. I., Terlevich, E., Pagel, B. E. J., Vilchez, J. M., \& Edmunds, M. G. 1987, MNRAS, 226, 19

Dopita, M. A., \& Evans, I. N. 1986, ApJ, 307, 431

Edmunds, M. G., \& Pagel, B. E. J. 1984, MNRAS, 211, 507

Esteban, C., Peimbert, M., Torres-Peimbert, S., \& Escalante, V. 1998, MNRAS, 295, 401

Esteban, C., Peimbert, M., Torres-Peimbert, S., \& GarciaRojas, J. 1999a, Rev. Mex. Astron. Astrofis., 35, 65

Esteban, C., Peimbert, M., Torres-Peimbert, S., Garcia-Rojas, J., \& Rodriguez, M. 1999b, ApJSS, 120, 113

Garnett, D. R. 1992, AJ, 103, 1330

Garnett, D. R., Shields, G. A., Skillman, E. D., Sagan, S. P., \& Dufour, R. J. 1997, ApJ, 489, 63

Izotov, Y. I., \& Thuan, T. X. 1998, ApJ, 500, 188

Izotov, Y. I., \& Thuan, T. X. 1999, ApJ, 511, 639

Izotov, Y. I., Thuan, T. X., \& Lipovetsky, V. A. 1994, ApJ, 435,647

Izotov, Y. I., Thuan, T. X., \& Lipovetsky, V. A. 1997, ApJSS, 108,1

Kaler, J. B. 1978, ApJ, 220, 887

Kennicutt, R. C., Bresolin, F., French, H., \& Martin, P. 2000, ApJ, 537, 589

Kobulnicky, H. A., \& Skillman, E. D. 1996, ApJ, 471, 211

Kobulnicky, H. A., \& Skillman, E. D. 1997, ApJ, 489, 636

Kobulnicky, H. A., \& Skillman, E. D. 1998, ApJ, 497, 601

Kobulnicky, H. A., Skillman, E. D., Roy, J.-R., Walsh, J. R., \& Rosa, M. R. 1997, ApJ, 477, 679

McCall, M. L., Rybski, P. M., \& Shields, G. A. 1985, ApJSS, 57,1

McGaugh, S. S. 1991, ApJ, 380, 140

Oey, M. S., \& Shields, J. C. 2000, ApJ, 539, 706

Oey, M. S., Dopita, M. A., Shields, J. C., \& Smith, R. C. 2000, ApJSS, 128, 511

Pagel, B. E. J., Edmunds, M. G., Blackwell, D. E., Chun, M. S., \& Smith, G. 1979, MNRAS, 189, 95

Pagel, B. E. J., Edmunds, M. G., \& Smith, G. 1980, MNRAS, 193, 219

Pagel, B. E. J., Simonson, E. A., Terlevich, R. J., \& Edmunds, M. G. 1992, MNRAS, 255, 325

Pilyugin, L. S. 2000, A\&A, 362, 325 (Paper I)

Pilyugin, L. S., \& Ferrini F. 1998, A\&A, 336, 103

Pilyugin, L. S., \& Ferrini, F. 2000, A\&A, 354, 874

Shaver, P. A., McGee, R. X., Newton, L. M., Danks, A. C., \& Pottasch, S. R. 1983, MNRAS, 204, 53

Skillman, E. D., Terlevich, R. J., Kennicutt, R. C. Jr., Garnett, D. R., \& Terlevich, E. 1994, ApJ, 431, 172

Smith, H. E. 1975, ApJ, 199, 591

Sobolev, V. V. 1967, Course of Theoretical Astrophysics, Moscow, Nauka

Stasinska, G. 1990, A\&ASS, 83, 501

Stasinska, G. 2000, New Astron. Rev., 44, 275

Torres-Peimbert, S., Peimbert, M., \& Fierro, J. 1989, ApJ, 345, 186

van Zee, L., Haynes, M. P., \& Salzer, J. J. 1997, AJ, 114, 2479

van Zee, L., Salzer, J. J., Haynes, M. P., O’Donoghue, A. A., \& Balonek, T. J. 1998, AJ, 116, 2805

Vilchez, J. M., \& Pagel, B. E. J. 1988, MNRAS, 231, 257

Vilchez, J. M., Pagel, B. E. J., Diaz, A. I., Terlevich, E., \& Edmunds, M. G. 1988, MNRAS, 235, 633

Webster, B. L., \& Smith, M. G. 1983, MNRAS, 204, 743

Zaritsky, D., Kennicutt, R. C., Jr., \& Huchra, J. P. 1994, ApJ, 420,87 